\title{
Ex vivo decontamination of yeast-colonized dentures by iodine-thiocyanate complexes
}

This article was published in the following Dove Press journal:

Clinical, Cosmetic and Investigational Dentistry

\author{
Sarra Sebaa ${ }^{1,2}$ \\ Maxime Faltot ${ }^{1}$ \\ Sandra De Breucker ${ }^{3}$ \\ Zahia Boucherit-Otmani² \\ Françoise Bafort ${ }^{4}$ \\ Jean-Paul Perraudin ${ }^{5}$ \\ Philippe Courtois' \\ 'Laboratory of Physiology and \\ Pharmacology, Université Libre \\ de Bruxelles, Brussels, Belgium; \\ ${ }^{2}$ Laboratory of Antibiotics and \\ Antifungals: Physico-Chemistry, \\ Synthesis and Biological Activity, \\ University of Tlemcen, Tlemcen, \\ Algeria; ${ }^{3}$ Department of Geriatrics, \\ CUB - Hôpital Erasme, Brussels, \\ Belgium; ${ }^{4}$ Integrated and Urban \\ Plant Pathology Laboratory, Liège \\ University, Gembloux, Belgium; \\ ${ }^{5}$ Taradon Laboratory, Tubize, Belgium
}

Correspondence: Philippe Courtois Laboratory of Physiology and Pharmacology (CP 604), Université Libre de Bruxelles (ULB), Route de Lennik 808, B-I 070 Brussels, Belgium Email philippe.courtois@ulb.ac.be
Introduction: Under well-defined experimental conditions, and in the presence of hydrogen peroxide, lactoperoxidase produces stable iodine-thiocyanate complexes that have antimicrobial properties. A novel process was developed to short circuit the consumption of hydrogen peroxide by microbial catalases by producing iodine-thiocyanate complexes prior to contact with microorganisms, with the aim of being able to decontaminate the ex vivo dentures colonized by yeasts. Materials and methods: Teabags containing lactoperoxidase adsorbed on inert clay beads were immersed for 1 minute in phosphate buffer solution ( $0.1 \mathrm{M} \mathrm{pH} 7.4)$ containing $5.2 \mathrm{mM}$ potassium iodide, $1.2 \mathrm{mM}$ potassium thiocyanate, and $5.5 \mathrm{mM}$ hydrogen peroxide. After removing the adsorbed lactoperoxidase, the stability and efficacy of iodine-thiocyanate complexes for Candida-colonized denture decontamination were verified. Investigations were performed in vitro on Candida albicans ATCC 10231 and on clinical isolates from 46 dentures. A Candida plate count was performed after a 24-hour incubation at $37^{\circ} \mathrm{C}$ on Sabouraud-chloramphenicol or CHROMagar solid media; then, the yeast growth was evaluated in Sabouraud broth by turbidimetry and biofilm biomass by crystal violet staining.

Results: In vitro tests demonstrated the effectiveness of the oxidant solution in sterilizing a suspension of $10^{6}$ Candida cells per milliliter after a 5-minute incubation. A single ex vivo immersion of contaminated dentures in a solution of iodine-thiocyanate complexes led to a decrease of at least $1 \log$ unit in the number of colony-forming units in $58.3 \%$ of the tested dentures, while immersing in water alone had no effect on denture colonization (significant $\chi^{2}: p=0.0006$ ).

Conclusion: These data suggest a promising new strategy for decontamination of dentures.

Keywords: biofilm, Candida, hygiene, lactoperoxidase, oral cavity

\section{Introduction}

Peroxidases have been known for several decades as antimicrobial factors in the oral environment. ${ }^{1-4}$ They originate from the salivary glands (sialoperoxidases) or from neutrophils (myeloperoxidase) passing into the oral cavity through the gingival sulci. In the presence of hydrogen peroxide $\left(\mathrm{H}_{2} \mathrm{O}_{2}\right)$, both enzymes convert salivary thiocyanate $\left(\mathrm{SCN}^{-}\right)$into hypothiocyanite $\left(\mathrm{OSCN}^{-}\right)$, which is capable of inhibiting different oral bacterial species (among others Streptococcus mutans, Lactobacillus acidophilus, and black-pigmented anaerobes) and yeasts such as Candida albicans. 5,6 In vitro, these peroxidases are also capable of oxidizing iodide $\left(\mathrm{I}^{-}\right)$to hypoiodite $\left(\mathrm{OI}^{-}\right)$; the lactoperoxidase from bovine milk also shows the ability to form $\mathrm{OSCN}^{-}$or $\mathrm{OI}^{-}{ }^{-7,8}$ In tissues, myeloperoxidase forms hypochlorite $\left(\mathrm{OCl}^{-}\right)$from the chloride ion $\left(\mathrm{Cl}^{-}\right)$. Based on promising in vitro experimental data, hypohalous $\left(\mathrm{OI}^{-}\right)$or pseudohypohalous 
$\left(\mathrm{OSCN}^{-}\right)$compounds have been suggested as adjuvants to improve oral hygiene or to decontaminate dentures colonized by Candida. For this reason, lactoperoxidase has been incorporated into the oral hygiene products with thiocyanate and a hydrogen peroxide donor often consisting of a glucose/ glucose oxidase enzyme sequence. However, the know-how transfer from in vitro to in vivo conditions has led to inconclusive data. ${ }^{9-11}$ The resulting paradox that is active in vitro, and inactive or minimally active in vivo, could be explained by the competition between oral peroxidases and microbial catalase for the same substrate, hydrogen peroxide. ${ }^{12}$ In fact, the hydrogen peroxide secreted in the oral environment by the salivary glands or produced by neutrophils and some microbial species can be immediately consumed either by peroxidases or by enzymes such as catalase or glutathione peroxidase. The presence of $\mathrm{H}_{2} \mathrm{O}_{2}$-detoxifying enzymes helps reduce hypohalous oral concentrations, thus avoiding reaching the antimicrobial levels and further regulating the oral microflora. Ex vivo use for decontamination of dentures can short circuit the competition for $\mathrm{H}_{2} \mathrm{O}_{2}$ due to detoxifying enzymes in the oral environment; hence, the antimicrobial effect on the microbial flora depends on the balance between the enrichment in oxidants and the catalase activity present in the biofilms attached to the denture. Recent in vitro studies evaluated the effects of both iodine and thiocyanate when simultaneously incorporated into a peroxidase system on phytopathogens such as Penicillium expansum..$^{13}$ Under well-defined experimental conditions, and in the presence of hydrogen peroxide, lactoperoxidase produces stable iodine-thiocyanate complexes such as $\mathrm{I}_{2}(\mathrm{SCN})^{-}$, which have antimicrobial properties. ${ }^{14}$

The aim of this study was to validate a new procedure for the production of iodine-thiocyanate complexes by lactoperoxidase adsorbed on a removable support that could be used to clean a Candida-contaminated denture ex vivo. In this study, the peroxidase-produced oxidants were tested in vitro, first on the reference strain C. albicans ATCC 10231 (often used for the evaluation of antifungal molecules), then on clinical strains isolated by swabbing resin dentures. Finally, an ex vivo clinical trial was performed to confirm the data obtained in vitro.

\section{Materials and methods Microorganisms}

Yeasts were aerobically grown at $37^{\circ} \mathrm{C}$ for 24 hours in Sabouraud broth (OXOID ${ }^{\text {TM }}$ CM147; Basingstoke, UK) or on solid medium, such as Sabouraud agar with chloramphenicol and gentamicin or CHROMagar ${ }^{\mathrm{TM}}$ media (BD Diagnostics ${ }^{\mathrm{TM}}$, Erembodegem, Belgium). The investigations were carried out on C. albicans ATCC 10231 (Culti-Loops $^{\text {TM, Thermo }}$ Scientific $^{\mathrm{TM}}$, Lenexa, KS, USA), which is often chosen to test antifungals, ${ }^{15}$ and on clinical strains sampled from dentures using sterile cotton swabs (EUROTUBO ${ }^{\circledR}$; Deltalab ${ }^{\mathrm{TM}}$, Barcelona, Spain). Clinical isolates were identified according to the appearance of their colonies on CHROMagar ${ }^{\mathrm{TM}}$ medium, by chlamydoconidia formation on Rice Extract agar with Polysorbate 80 (BD Diagnostics ${ }^{\mathrm{TM}}$ ) and using the API yeast identification system (bioMérieux, Marcy-1'Etoile, France). All in vitro investigations were performed in a third subculture on Sabouraud solid medium. Candida suspensions were adjusted in phosphate buffer $\left(0.1 \mathrm{M} \mathrm{pH} \mathrm{7.4)} \mathrm{to} 10^{6}\right.$ (or $2 \times 10^{7}$ ) blastoconidia per $\mathrm{mL}$ by dilution after counting in a Thoma cell chamber (Marienfeld ${ }^{\mathrm{TM}}$, Lauda-Königshofen, Germany).

\section{Oxidant production}

$\mathrm{H}_{2} \mathrm{O}_{2}\left(\right.$ Perhydrol $\left.^{\circledR}\right)$, potassium iodide (KI), and potassium thiocyanate $(\mathrm{KSCN})$ were purchased from Merck ${ }^{\mathrm{TM}}$ (Darmstadt, Germany). A teabag containing $50 \mathrm{mg}$ lactoperoxidase (Taradon, Tubize, Belgium) and $100 \mathrm{mg}$ inert clay beads was immersed in $1 \mathrm{~L}$ phosphate buffer $(0.1 \mathrm{M}$ $\mathrm{pH}$ 7.4). After dissolution of KI (5.2 $\mathrm{mM}$ final) and $\mathrm{KSCN}$ (1.2 mM final), $550 \mu \mathrm{L}$ of Perhydrol ${ }^{\circledR}$ (5.5 mM final) was added dropwise with stirring for 1 minute. The bag was then removed and the solution was stored at $4{ }^{\circ} \mathrm{C}$. Before use, the oxidative compounds produced by the aforementioned procedure were assayed using a dithiobisnitrobenzoic acid (DTNB) spectrophotometric method adapted from Aune and Thomas (1977). ${ }^{16}$ After reduction of $146 \mu \mathrm{M}$ DTNB (Sigma-Aldrich $^{\mathrm{TM}}$, St Louis, MO, USA) using $142 \mu \mathrm{M}$ mercaptoethanol (Merck ${ }^{\mathrm{TM}}$ ), thionitrobenzoic (TNB) acid reagent (total volume of $2 \mathrm{~mL}$ ) was mixed with the solution containing the oxidants (from 0 to $500 \mu \mathrm{L}$ made up to $500 \mu \mathrm{L}$ with $0.1 \mathrm{M}$ phosphate buffer, $\mathrm{pH}$ 7.4). The TNB that absorbs light at $412 \mathrm{~nm}$ was then oxidized to the colorless DTNB. According to the Beer-Lambert law (molar extinction coefficient $\varepsilon$ equal to $13,600 \mathrm{M}^{-1} \cdot \mathrm{cm}^{-1}$ ), the number of moles of oxidized TNB was calculated and transformed into oxidant concentration presuming that one mole of oxidant oxidizes two moles of TNB. Figure 1 shows the analytical range for determining the oxidant concentration by using normalized data from four independent experiments. Between 8.4 and $75.3 \mu \mathrm{M}$ of oxidant, absorbance at $412 \mathrm{~nm}$ is proportional to the concentration: the relation is then linear $(\mathrm{y}=0.014 \mathrm{x}$, $r=0.9930$ ). The inter-day precision of the method was $9.3 \%$. The inset in Figure 1 represents the experimental absorbance after mixing different volumes of fresh oxidant solution with reagents. 


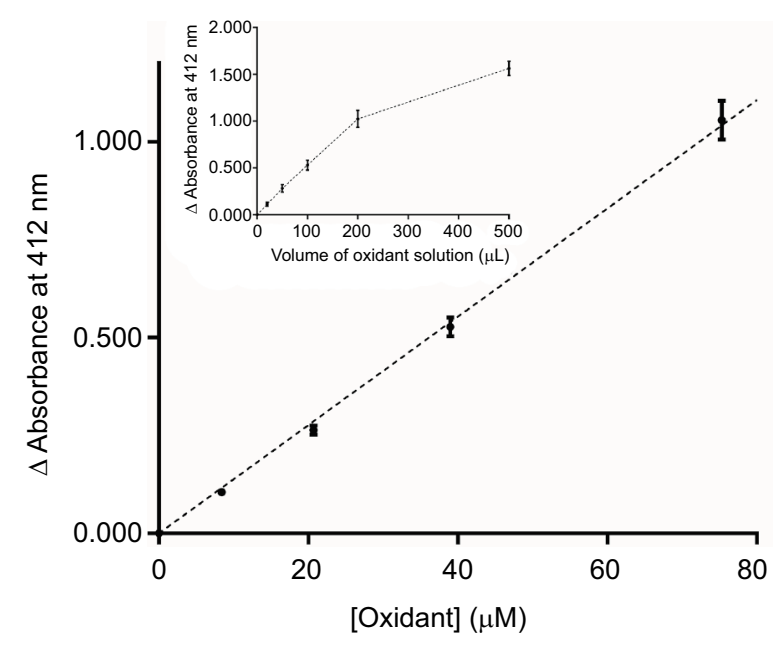

Figure I DTNB oxidation by iodine-thiocyanate complexes: relation between absorbance at $412 \mathrm{~nm}$ and oxidant concentration in the reaction medium (mean \pm SEM). Inset: relation between absorbance and oxidant solution volume transferred in the reaction medium. The various volumes were made up to $0.5 \mathrm{~mL}$ with phosphate buffer and then added to the same reagent volume $(2 \mathrm{~mL})$ to obtain a $2.5 \mathrm{~mL}$ final volume.

Abbreviations: DTNB, dithiobisnitrobenzoic acid; SEM, standard error of the mean.

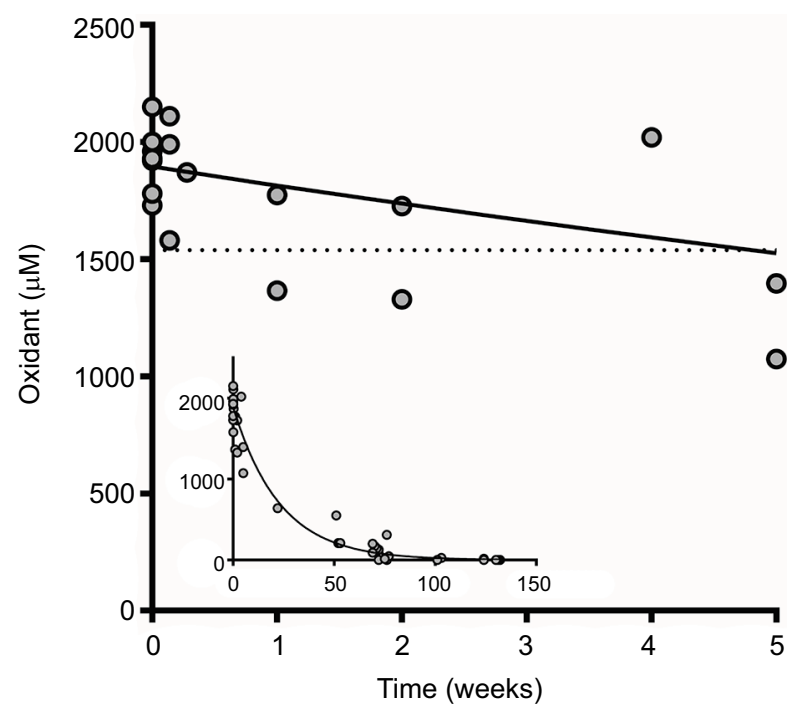

Figure 2 Evaluation of iodine-thiocyanate complexes concentration in solutions stored at $4^{\circ} \mathrm{C}$ (average of 4 replicates for each of the solutions tested). Oxidant production was assayed by the DTNB method in 47 different oxidant solutions after variable storage times (from I day to I 32 weeks). Initial concentrations were 1929 $\pm 46 \mu \mathrm{M}$ (mean \pm SEM, $n=8$ ). Dotted line indicates the value corresponding to the mean -3 SD of the initial concentrations.

Abbreviations: DTNB, dithiobisnitrobenzoic acid; SEM, standard error of the mean.

Figure 2 shows the concentration of oxidants found after different storage times at $4^{\circ} \mathrm{C}$ (from 1 day to 132 weeks). Initial concentrations were $1929 \pm 46 \mu \mathrm{M}$ (mean \pm standard error of the mean $[\mathrm{SEM}], \mathrm{n}=8$ ): the coefficient of variation
(CV) was $6.7 \%$. These levels appear to slowly decrease over 4 weeks and then decayed exponentially $(r=0.9751)$ to reach zero after 50 weeks. After 5 weeks of storage at $4^{\circ} \mathrm{C}$, the concentration of iodine-thiocyanate complexes had decreased by $20.9 \%$ of the initial value; at 50 weeks of storage at $4{ }^{\circ} \mathrm{C}$, the concentration of iodine-thiocyanate complexes had decreased by $88.8 \%$. In the context of this investigation, the oxidant concentration was always determined on the day of use.

\section{Candidacidal effect}

The candidacidal effect of the solution of iodine-thiocyanate complexes was evaluated by counting colony-forming units (CFUs) on Petri dishes for the reference strain ATCC 10231. The suspensions of C. albicans $\left(10^{7}\right.$ or $2 \times 10^{8}$ blastoconidia in $0.1 \mathrm{~mL}$ of $0.1 \mathrm{M}$ phosphate buffer, $\mathrm{pH} 7.4$ ) were incubated for 5 minutes in the presence of oxidizing solution $(9.9 \mathrm{~mL})$. A control suspension was incubated in phosphate buffer alone. In addition, 3 different conditions were simultaneously tested on the yeast suspensions: distilled $\mathrm{H}_{2} \mathrm{O}, 5.5 \mathrm{mM} \mathrm{H}_{2} \mathrm{O}_{2}$ in buffer and a solution prepared without $\mathrm{H}_{2} \mathrm{O}_{2}$ thus containing only $1.2 \mathrm{mM} \mathrm{KSCN}$, and $5.2 \mathrm{mM} \mathrm{KI}$ substrates in buffer. After incubation, $20 \mu \mathrm{L}$ (from undiluted test tubes and from 100-fold diluted control tubes) was spread on Sabouraud agar. Colonies were counted after 24 hours of incubation at $37^{\circ} \mathrm{C}$ using an e-Count ${ }^{\mathrm{TM}}$ colony counter (Heathrow Scientific, Vernon Hills, IL, USA). Oxidant concentration was determined by spectrophotometry, and C. albicans ATCC 10231 sensitivity to the oxidant was measured in each solution to check in vitro efficiency before further investigations and clinical trials.

\section{Biofilm production and quantification}

Candida biofilms were produced in flat-bottom 96-well (12 columns of 8 wells) polystyrene plates (GreinerBio-one ${ }^{\circledR}$, CellStar ${ }^{\mathrm{TM}}$, Neuburg, Germany) for reference and 6 clinical strains randomly taken from denture swabs. Each column of the microplate corresponded to an experimental condition repeated 8 times. The minimal inhibitory concentration of iodine-thiocyanate complexes was determined by evaluation of turbidimetric growth in broth and by quantification of attached biomass in the reactive wells for the reference strain and for 6 clinical isolates from dentures. Yeast growth and their capacity to form biofilms were evaluated after 24 hours at $37^{\circ} \mathrm{C}$ in flat-bottom 96-well plates in liquid Sabouraud medium with increasing concentrations of oxidants ranging from 31 to $1000 \mu \mathrm{M}$. Using Combitips pipettes or a MultiChannel pipette (Eppendorf ${ }^{\circledR}$, Germany), each well 
initially contained $0.5 \times 10^{6}$ blastoconidia suspended in $25 \mu \mathrm{L}$ of $0.1 \mathrm{M}$ phosphate buffer at $\mathrm{pH}$ 7.4. After adding the same volume $(25 \mu \mathrm{L})$ of oxidizing solution, the plate was mixed for 5 minutes at room temperature using a magnetic stirrer (Ikamag, Boutersem, Belgium). Sabouraud broth was added (final volume of each well: $250 \mu \mathrm{L}$ ). After a 24-hour incubation at $37^{\circ} \mathrm{C}$, the growth of the yeast was evaluated photometrically at $600 \mathrm{~nm}$ using a microplate reader (Packard SpectraCount ${ }^{\mathrm{TM}}$; PerkinElmer, Wellesley, MA, USA). This turbidimetric measurement indifferently evaluates the planktonic phase and the phase attached to the bottom of the well plus sedimented cells. The data were compared with a control without the oxidants and a sterility control without the cell suspension, which confirmed the absence of accidental contamination during handling. Turbidimetric measurement in a series of 8 ATCC-control replicates showed a CV between $1.7 \%$ and $6.6 \%$. After washing, the biofilms were quantified by crystal violet staining. ${ }^{17}$ After opacimetric reading at 24 hours, the culture medium was carefully aspirated using a MultiChannel pipette (VWR ${ }^{\mathrm{TM}}$, Leuven, Belgium). The wells of the microplate were washed 3 times with a $0.9 \% \mathrm{NaCl}$ solution in order to eliminate the non-adherent cells. The attached biomass was fixed with methanol (Sigma-Aldrich ${ }^{\mathrm{TM}}$ ) for 15 minutes at room temperature and stained with $2 \%$ filtered crystal violet ( $8 \mathrm{~g}$ of crystal violet in $80 \mathrm{~mL}$ of ethanol $95 \%$ plus $3.2 \mathrm{~g}$ of ammonium oxalate in $320 \mathrm{~mL}$ of distilled water) for 5 minutes at room temperature. After rinsing with tap water and drying, the colored biomass fixed on the walls of the microplate wells was solubilized with $2 \mathrm{M}$ acetic acid and absorbance was photometrically measured at $600 \mathrm{~nm}$. The precision and accuracy of the absorbance measured using the Packard SpectraCount ${ }^{\mathrm{TM}}$ Microplate Reader is acceptable up to an optical density of 2,500 (CV and coefficient of accuracy $<5 \%$ ). However, all samples with an absorbance value higher than 2,000 were diluted 10-fold. Measurement of crystal violet absorbance in series of 8 ATCC-control replicates showed a CV between $10.9 \%$ and $29.5 \%$.

Acrylic resin (Palapress ${ }^{\circledR}$; Kulzer, Hanau, Germany) pieces $(3 \times 18 \times 5 \mathrm{~mm})$ were produced as previously described and stored at $4{ }^{\circ} \mathrm{C}$ in $0.1 \%(\mathrm{w} / \mathrm{v})$ sodium azide. ${ }^{18}$ On the day of the experiment, resin pieces were washed for 5 minutes at room temperature, 3 times with $60 \mathrm{~mL}$ sterile distilled water, and once with $60 \mathrm{~mL}$ Sabouraud liquid medium. For biofilm production on this support, two pieces were transferred to $15 \mathrm{~mL}$ conical Falcon tubes made of polypropylene (BD Biosciences, Erembodegem, Belgium) and immersed in $4 \mathrm{~mL}$ Sabouraud medium containing a $C$. albicans ATCC 10231 suspension $\left(10^{6}\right.$ blastoconidia/mL). After a 24-hour incubation at $37^{\circ} \mathrm{C}$, the liquid phase was carefully aspirated and its turbidity measured at $600 \mathrm{~nm}$. A control without yeast attested to the absence of contamination during the process. Resin pieces were then transferred into new sterile Falcon tubes by sliding and were washed 3 times in sterile phosphate buffer (0.1 M, pH 7.4). Finally, they were immersed for 30 minutes in the solution of iodine-thiocyanate complexes or in phosphate buffer as a control, both supplemented with glucose $(2 \mathrm{~g} / 100 \mathrm{~mL})$. Swabs from each surface were seeded onto Sabouraud (with $0.4 \mathrm{~g} / \mathrm{L}$ chloramphenicol and $0.04 \mathrm{~g} / \mathrm{L}$ gentamycin) solid medium for a CFU count performed after 48 hours at $37^{\circ} \mathrm{C}$.

\section{Dentures}

Denture wearers were recruited at the Erasme Hospital (ULB, Brussels) from the dentistry or geriatrics departments between January and March 2016. The protocol was approved on October 22, 2015, by the Erasme-ULB Ethics Committee under number P2015/413; the participants provided written informed consent for their data to be anonymously published. The recruited denture wearers had a healthy oral mucosa free of any clinical oral infection, namely of prosthetic stomatitis or other mucosal candidiasis. Enrolled patients $(\mathrm{N}=46$, between 41 and 98 years old) were alternately allocated to a test or control group of 23 patients each. All participants completed a questionnaire on the denture characteristics and their hygiene habits. The ex vivo treatment of dentures was carried out in 3 steps in a single session: 1) swabbing (time zero) of the denture resin (palatin side) to detect the presence of Candida, 2) immersing the denture for 5 minutes in the iodine-thiocyanate complexes solution (test group) or sterile water (control group) followed by rinsing with sterile water, and 3 ) reswabbing of denture to detect the residual presence of Candida. Moreover, the dentures in the control group were immersed in the solution of iodine-thiocyanate complexes, and then swabbed 3 times. At this point, the clinician was not aware of the dentures that were contaminated with Candida.

The swabs were immediately seeded on ChromAgar $\AA$ agar for the Candida plate count and species diagnostics. Subcultures were stored at $4^{\circ} \mathrm{C}$ and all in vitro investigations were performed on a third subculture that was started the day before each experiment.

\section{Toxicity of oxidants}

The toxicity of the oxidant solution was evaluated using the Trypan blue method on oral epithelial cells present in saliva after desquamation. Trypan blue that enters living cells is immediately expelled: the living cells do not have a colored 
appearance whereas the dead cells appear colored in blue. The saliva of 6 adults ( 5 women and 1 man aged 25-65 years) was collected in sterile containers (Deltalab ${ }^{\mathrm{TM}}$ ). A volume of $50 \mu \mathrm{L}$ of saliva diluted twice (in $0.9 \% \mathrm{NaCl}$ ) was mixed with $50 \mu \mathrm{L}$ of a Trypan blue solution (Merck ${ }^{\mathrm{TM}}$ ) at a concentration of $0.4 \mathrm{~g} / 100 \mathrm{~mL}$. The percentage of dead cells (on a total of at least 120 cells in each preparation) was observed between a slide (Thermo Scientific ${ }^{\mathrm{TM}}$, Braunschweig, Germany) and coverslip (Marienfeld, Lauda-Königshofen, Germany) on a Leica DM2000 optical microscope (Leica ${ }^{\mathrm{TM}}$, Wetzlar, Germany). A 30-minute incubation (room temperature) of 100 $\mu \mathrm{L}$ of saliva with $100 \mu \mathrm{L}$ of iodine-thiocyanate complexes solution or $0.4 \%(\mathrm{w} / \mathrm{v}$ in phosphate buffer) chlorhexidine digluconate (Sigma-Aldrich ${ }^{\mathrm{TM}}$ ) preceded the microscopic examination to check the toxicity of these compounds.

\section{Statistics}

Experimental data were normalized in percentage by dividing each experimental value by the arithmetic mean of the control values obtained in the same experiment. Mean values are expressed with their related SEM, unless indicated otherwise. Statistical tests (average, standard deviation, SEM, Kolmogorov-Smirnov test, unpaired Student's $t$-test, analysis of variance supplemented by a Dunnett's multiple comparison post-test, chi-square test) were performed using GraphPadPrism software, version 7.01, for Windows (GraphPad Software, San Diego California USA; www.graphpad.com). This same software was used to carry out the mathematical analysis of the data and to draw graphs.

\section{Results \\ Candida susceptibility to iodine- thiocyanate complexes}

Figure 3 shows the effect of the various solution components, combined or not, on the survival rate of C. albicans ATCC 10231. Only the complete system had a candidacidal effect after a 5-minute incubation in the presence of $10^{6}$ Candida cells/mL. Neither thiocyanate/iodide substrates after incubation with the peroxidase enzyme nor $\mathrm{H}_{2} \mathrm{O}_{2}$ alone reduced the Candida CFU count. The survival rate of yeast in water did not differ from that observed in the buffer.

When tested on higher blastoconidia concentrations, the solution of iodine-thiocyanate complexes reduced the viability of Candida cells at least up to $2 \times 10^{7}$ blastoconidia/ $\mathrm{mL}$ in a 5-minute incubation (data not shown).

Figure 4 shows the effect of different oxidant concentrations (from 31 to $1000 \mu \mathrm{M}$ ) on the growth of Candida (reference strain and clinical isolates) in the wells of a

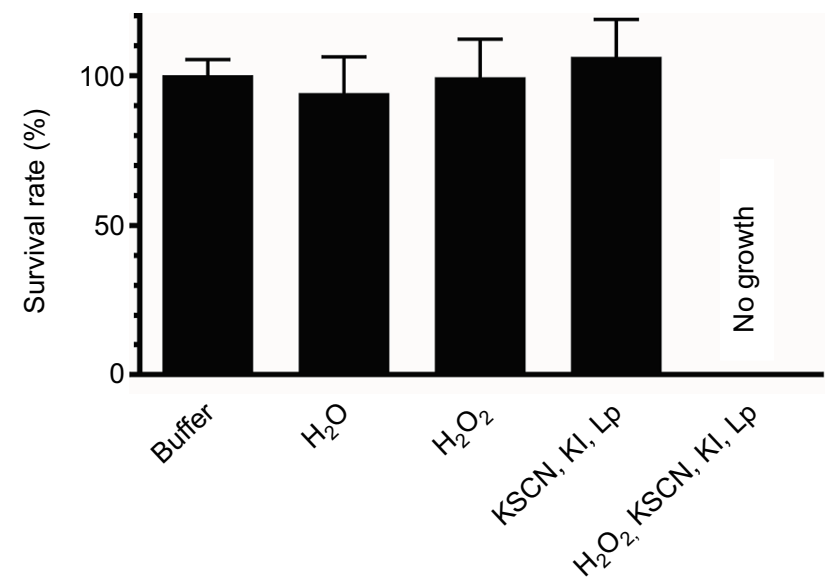

Figure 3 Candida inhibition after a 5-minute incubation at room temperature by various combinations of reagents used to form iodine-thiocyanate complexes. Candida albicans ATCC 10231 survival rate was evaluated by counting colonyforming units (CFUs) after cultivation on Sabouraud agar. Lp was clay-adsorbed inside a teabag. Data are expressed as the percentage of CFUs counted after incubation in $0.1 \mathrm{M}$ phosphate buffer $(\mathrm{pH} 7.4)$. Error bars indicate the standard error of the mean $(\mathrm{N}=4)$.

Abbreviation: Lp, lactoperoxidase; $\mathrm{H}_{2} \mathrm{O}_{2}$, hydrogen peroxide; $\mathrm{KSCN}$, potassium thiocyanate; $\mathrm{KI}$, potassium iodide.

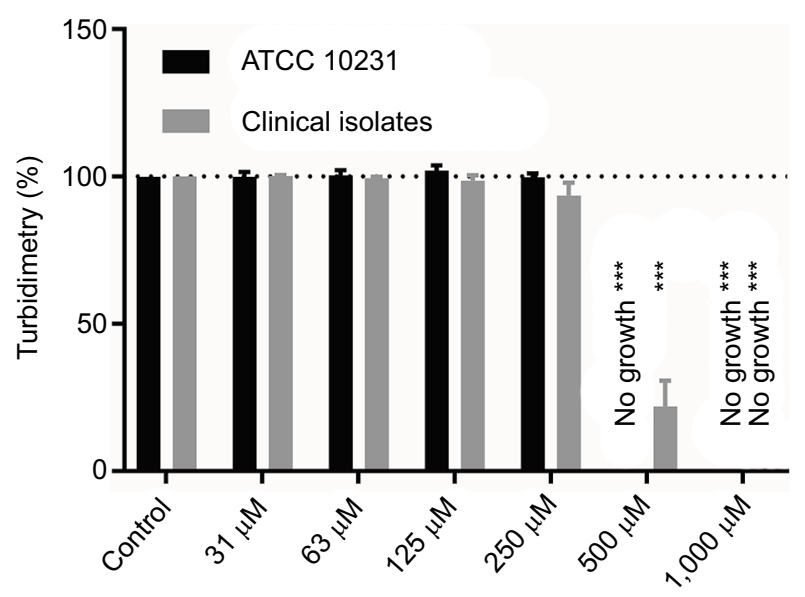

Figure 4 Effect of different concentrations of iodine-thiocyanate complexes on Candida albicans growth (ATCC 10231 and 6 clinical isolates) after a 5-minute incubation at room temperature in the wells of a 96 -well polystyrene plate. Yeast growth was then evaluated after addition of Sabouraud broth and incubation at $37^{\circ} \mathrm{C}$ for 24 hours. Data are expressed as the percentage of turbidity observed in the same conditions after incubation in $0.1 \mathrm{M}$ phosphate buffer ( $\mathrm{pH}$ 7.4). The different oxidant concentrations were compared with the paired control by ANOVA complemented by a Dunnett's multiple comparison test $(* * * p<0.00$ I). Error bars indicate the standard error of the mean $(\mathrm{N}=6)$.

Abbreviation: ANOVA, analysis of variance.

96-well polystyrene plate. The data from each independent experiment were expressed as the percentage of the paired controls conducted in the same plate, allowing us to estimate the variability of the results within each condition with respect to the day-control and to avoid the bias of variability between experiments. After a 24-hour incubation in the presence of $C$. albicans ATCC 10231, the mean turbidity 
of the culture medium in the control wells corresponded to an absorbance at $600 \mathrm{~nm}$ of $0.783 \pm 0.023(\mathrm{~N}=6)$. In 6 independent experiments, concentrations of iodine-thiocyanate complexes $\leq 250 \mu \mathrm{M}$ did not modify the turbidity observed in the wells when compared to the average of the paired controls. The absorbance at $600 \mathrm{~nm}$ after 24 hours of incubation at $37^{\circ} \mathrm{C}$ then varied between $99.8 \% \pm 1.3 \%$ and $102.1 \% \pm 1.7 \%$ of that observed for the paired controls (Dunnett, NS). A iodine-thiocyanate complex concentration $\geq 500 \mu \mathrm{M}$ significantly suppressed the growth evaluated by turbidimetry (Dunnett: $p<0.001$ ). The results obtained on clinical strains isolated from the dentures were similar. The mean turbidity of the culture medium in the control wells corresponded to an absorbance at $600 \mathrm{~nm}$ of $0.885 \pm 0.024$ $(\mathrm{N}=6)$. In the presence of iodine-thiocyanate complexes at a concentration $\leq 250 \mu \mathrm{M}$, the $600 \mathrm{~nm}$ absorbance after a 24 -hour incubation at $37^{\circ} \mathrm{C}$ ranged from $93.6 \% \pm 4.4 \%$ to $100.1 \% \pm 0.4 \%$ of that observed for paired controls (Dunnett, NS). Higher oxidant concentrations (500 and 1,000 $\mu \mathrm{M}$ ) significantly reduced growth by $78.1 \% \pm 8.8 \%$ and $99.4 \% \pm$ $0.6 \%$ of control growth, respectively (Dunnett, $p<0.001$ ).

Figure 5 evaluates the biomass attached to the inner surface of the wells of 96-well polystyrene plate after crystal violet staining for both the reference and clinical strains. After staining and 10-fold dilution, the controls showed an absorbance of $0.732 \pm 0.089(\mathrm{~N}=6)$ in ATCC 10231 strain while the clinical strains presented a mean absorbance com-

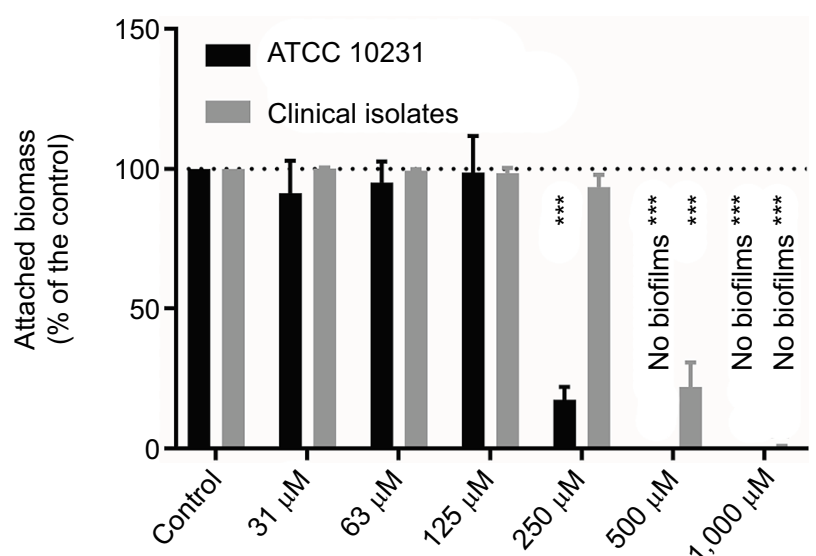

Figure 5 Effect of different concentrations of iodine-thiocyanate complexes on biofilm formation by Candida albicans strains (ATCC 1023I and 6 clinical isolates) after a 5-minute incubation at room temperature in the wells of 96-well polystyrene plate. Biofilm was then evaluated by staining the attached biomass after incubation at $37^{\circ} \mathrm{C}$ for 24 hours in Sabouraud broth. Data are expressed as the percentage of attached biomass observed in the same conditions after incubation in $0.1 \mathrm{M}$ phosphate buffer $(\mathrm{pH}$ 7.4). The different oxidant concentrations were compared with the paired control by ANOVA complemented by a Dunnett's multiple comparison test $\left({ }^{* * *} p<0.00 \mathrm{I}\right)$. Error bars indicate the standard error of the mean $(\mathrm{N}=6)$.

Abbreviation: ANOVA, analysis of variance. prised between 0.086 and 1.907 for their respective controls. For the ATCC strain, the results obtained in the presence of iodine-thiocyanate complexes at concentrations between 250 and 1,000 $\mu \mathrm{M}$ showed a statistically significant decrease (Dunnett: $p<0.001$ ). The inhibition was total at 500 and 1,000 $\mu \mathrm{M}$, whereas at $250 \mu \mathrm{M}$, the attached biomass corresponded to $17.4 \% \pm 4.6 \%(\mathrm{~N}=6)$ of that found for the paired controls. Again, the results obtained for the clinical strains were similar. Iodine-thiocyanate complexes at concentrations between 31 and $250 \mu \mathrm{M}$ did not modify the attached biomass observed in the wells compared to the average of the paired controls: the absorbance at $600 \mathrm{~nm}$ after 24 hours of incubation at $37^{\circ} \mathrm{C}$ ranged from $93.6 \% \pm 4.4 \%$ to $100.1 \% \pm 0.4 \%$ of that observed for controls (Dunnett, NS). Higher oxidant concentrations (500 and 1,000 $\mu \mathrm{M}$ ) significantly inhibited the biomass by $78.1 \pm 8.8 \%$ and $99.4 \% \pm 0.4 \%$ of the control biomass as assessed using the crystal violet method (Dunnett: $p<0.001$ ).

On the other hand, after 30 minutes of contact at room temperature, iodine-thiocyanate complexes completely abolished the presence of Candida biofilms on a resin piece contaminated with $C$. albicans ATCC 10231 as shown in Figure 6.

\section{Denture decontamination using iodine- thiocyanate complexes}

Table 1 details the number of patients recruited $(\mathrm{N}=46$ divided into 2 groups of 23 individuals) and enrolled after demonstration of Candida contamination $(\mathrm{N}=27 ; 12$ in the test group and 15 in the control group). The mean ages were 74.3 years for the control group $(\mathrm{N}=23$, median: 68.0 years $)$ and 79.5 years for the test group $(\mathrm{N}=23$, median: 82.0 years $)$. The mean ages of Candida-positive patients were 74.2 years for the control group $(\mathrm{N}=15)$ and 77.5 years for the test group $(\mathrm{N}=12)$. None of these patients suffered from oral mucosal disease, including prosthetic stomatitis. Hygiene techniques

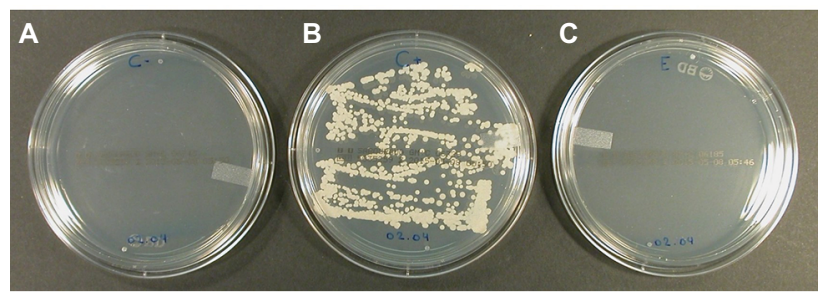

Figure 6 Sabouraud agar plates inoculated by swabbing contaminated acrylic resin pieces and incubated for 48 hours at $37^{\circ} \mathrm{C}$. The resin pieces were previously subjected to different processes. (A) Uncontaminated acrylic resin piece processed to attest the sterility of handling. (B) Contaminated (Candida albicans ATCC I0231) acrylic resin piece immersed for 30 minutes in phosphate buffer $(0.1 \mathrm{M}, \mathrm{pH} 7.4)$ at room temperature. (C) Contaminated (C. albicans ATCC I023I) acrylic resin piece immersed for 30 minutes in the solution of iodine-thiocyanate complexes at room temperature. The data are representative of 4 independent experiments. 
Table I Status of Candida contamination for investigated dentures

\begin{tabular}{lll}
\hline Number of cases & Test & Control \\
\hline Number of recruited denture wearers & 23 & 23 \\
Number of Candida-colonized dentures & 12 & 15 \\
$\quad$ Plurispecies colonization & 3 & 1 \\
$\quad$ Monospecies colonization & 9 & 14 \\
Number of enrolled Candida-contaminated & 12 & 15 \\
dentures & & \\
Number of recruited patients reporting using the procedure for \\
cleaning denture \\
$\begin{array}{l}\text { Brushing with water } \\
\text { Brushing with toothpaste }\end{array}$ \\
$\begin{array}{l}\text { Soaking in a commercial cleansing tablet } \\
\text { solution }\end{array}$ \\
$\begin{array}{l}\text { Soaking in a commercial mouthwash solution } \\
\text { Soaking in bleach }\end{array}$ & 18 & 4 \\
\hline
\end{tabular}

Note: Status of Candida contamination (prevalence, number of different species, and hygiene procedure used) in both investigated groups (test and control).

had no effect on the denture colonization by Candida. Indeed the denture wearers from both groups claimed to practice the different procedures in similar proportions $\left(\chi^{2}\right.$ test, $p=0.5003$, NS). The rate of denture colonization was $58.7 \%$ in the patients examined in this study $(\mathrm{N}=46)$. In $55.6 \%$ of the wearers with Candida contamination, denture swabbing returned more than $50 \mathrm{CFUs}$ per Petri dish. More than half of the dentures $(85.2 \%, \mathrm{~N}=23$ for a total of 27 wearers with a Candida-contaminated denture) showed a "monospecies" contamination (a single species of yeast based on the color of macroscopic colonies observed on CHROMagar ${ }^{\circledR}$ medium). The remaining $14.8 \%(\mathrm{~N}=4$ for a total of 27 edentulous patients) were colonized by several "species" ( 2 or 3 different yeast species). The albicans species was found in $74.1 \%$ of the positive cultures $(\mathrm{N}=27)$. The second most commonly identified species was $C$. glabrata $(18.5 \%)$. The association C. albicans plus C. glabrata was found in $11.1 \%$ of Candida positive patients.

Table 2 reports the results of the clinical trial. A single 5 -minute ex vivo immersion in the iodine-thiocyanate complexes resulted in a decrease of at least $1 \log$ unit in the number of CFUs in 7 out of 12 cases $(58.3 \%)$, whereas immersing in water had no effect on denture colonization. This effect was statistically significant $\left(\chi^{2}, p=0.0006\right.$ and Fisher's test, $p=0.0009$ ). A decrease of at least $1 \log$ unit corresponds to a decrease of more than $90 \%$ of the CFUs initially counted. The dental prostheses of the control group were also submitted to a final immersion of 5 minutes in the iodine-thiocyanate complexes and then to reswabbing: $60 \%$ of the control dentures then showed a decrease of at least 1 logarithmic unit. This confirmed the data observed in the
Table 2 Ex vivo denture decontamination by iodine-thiocyanate complexes

\begin{tabular}{lll}
\hline A & Test & Control \\
\hline $\begin{array}{l}\text { Reduction of at least I logarithmic unit } \\
\begin{array}{l}\text { No reduction or reduction by less than I } \\
\text { logarithmic unit }\end{array}\end{array}$ & 7 & 0 \\
\hline B & & 15 \\
\hline Number of control dentures rinsed & First in & Then in \\
& $\mathbf{H}_{2} \mathbf{O}$ & test \\
& & solution \\
\hline Reduction of at least I logarithmic unit & 0 & 9 \\
No reduction of at least I logarithmic unit & 15 & 6 \\
\hline
\end{tabular}

Notes: A: Effect of a 5-minute incubation at room temperature with iodinethiocyanate complexes (test group) compared with water (control group) on denture Candida-carriage. B: Effect of a supplementary 5-minute incubation at room temperature with lactoperoxidase-produced oxidants on Candida-carriage of control dentures after water treatment.

test group and gave these patients the same chance of having a decontaminated prosthesis in the context of this study. Moreover, in the test group, a general downward trend in the number of colonies present after soaking was apparent: a reduction in the Candida (whatever the species) count by half was observed in $83.3 \%$ of cases after a 5 -minute incubation in the oxidant solution. In the same manner, the trend was similar in the secondarily treated control group: $73.3 \%$ showing at least a halving of Candida plate count. The control solution (water) led to a decrease in the number of CFUs of at least half in $26.7 \%$ of the cases but never a decrease of the order of $1 \log$ unit. It should be noted too that the Candida CFU count showed an increase in the number of CFUs in $13.3 \%$ of cases after soaking in water.

\section{Toxicity of oxidants}

The percentage of Trypan blue-stained epithelial cells was determined in saliva from 6 adults, before and after addition of the oxidative solution. The effect of iodine-thiocyanate complexes on Candida growth and its ability to form biofilms revealed lower oral epithelial cell toxicity than that of chlorhexidine, an antiseptic that is widely used in stomatology. Indeed, Table 3 shows an increase in the number of epithelial cells stained by Trypan blue after 30 minutes of incubation at room temperature, lower than that found in the presence of $0.2 \%(\mathrm{w} / \mathrm{v})$ chlorhexidine under the same experimental conditions. An ANOVA supplemented by a Dunnett test showed that this increase was significant for the $1000 \mu \mathrm{M}$ oxidant solution $(p<0.001)$ and $0.2 \%(\mathrm{w} / \mathrm{v})$ chlorhexidine $(p<0.001)$.

\section{Discussion}

This study aimed to evaluate the effect of oxidants produced by peroxidase on an oral microorganism by avoiding interference from the components of the peroxidase system 
Table 3 Toxicity evaluation of oxidant solution

\begin{tabular}{|c|c|}
\hline Assay condition & $\begin{array}{l}\text { Trypan blue- } \\
\text { positive cells (\%) }\end{array}$ \\
\hline Control & $34.8 \pm 2.0(\mathrm{~N}=6)$ \\
\hline lodine-thiocyanate complex solution, $300 \mu \mathrm{M}$ & $4 I .8 \pm 1.0(\mathrm{~N}=3)$ \\
\hline lodine-thiocyanate complex solution, $1000 \mu \mathrm{M}$ & $54.8 \pm 6.0(\mathrm{~N}=3)^{* * *}$ \\
\hline Chlorhexidine $0.2 \%$ & $100.0 \pm 0.0(\mathrm{~N}=6)^{* * *}$ \\
\hline \multicolumn{2}{|c|}{$\begin{array}{l}\text { Notes: Percentage of saliva epithelial cells stained by Trypan blue after a } 30 \text {-minute } \\
\text { incubation in the presence of iodine-thiocyanate complexes }(300 \mathrm{vs} \mathrm{I}, 000 \mu \mathrm{M}) \\
\text { or chlorhexidine }(0.2 \%) \text {. The different conditions were compared with the paired } \\
\text { control by ANOVA complemented by a Dunnett's multiple comparison test } \\
(* * * p<0.00 \mathrm{I}) \text {. }\end{array}$} \\
\hline
\end{tabular}

that generate them. Indeed, the new investigated process bypassed the presence, in the final solution, of enzymatic proteins (potentially immunogenic for in vivo applications) and the presence of hydrogen peroxide, one of the peroxidase substrates that is unstable and which is itself oxidizing. This study demonstrated the effectiveness of iodine-thiocyanate complexes produced by lactoperoxidase adsorbed on clay at inhibiting C. albicans (both from a reference source and clinical strains) in vitro. The prior preparation of iodine-thiocyanate complexes in the absence of (oral) microorganisms bypassed the consumption of hydrogen peroxide by microbial catalases. In addition, the presence of lactoperoxidase - without its substrates - could become a microbial nutrient and thus stimulate the growth of certain bacterial species in the oral cavity. ${ }^{19}$ The method already implemented in agronomy makes it possible to obtain lethal oxidant concentrations for C. albicans close to the concentrations of hypothiocyanite observed in whole saliva, namely up to $300 \mu \mathrm{M}{ }^{6,13}$ The iodine-thiocyanate complexes inhibited at least up to $2 \times 10^{7}$ blastoconidia/mL in a 5 -minute incubation. Even by reducing the concentration of the initial oxidizing solution (1929 $\pm 46 \mu \mathrm{M}$ ) to $500 \mu \mathrm{M}$, the inhibitory capacity in vitro remains efficient. After 5 weeks, the residual oxidizing power of the solution (ie, $80 \%$ of the initial oxidant concentration close to $2,000 \mu \mathrm{M}$ ) was still sufficient to inhibit a suspension of $10^{6}$ blastoconidia $/ \mathrm{mL}$. The iodine-thiocyanate complexes sterilized resin surfaces contaminated with more $C$. albicans than is usually found on patient dentures. These data suggest that this simple procedure could be used for the decontamination of dentures. The ease of processing these solutions will allow their use at home by edentulous patients. The stability of candidacidal activity during storage is another advantage.

\section{Combining thiocyanate and iodide substrates}

Both peroxidase substrates, thiocyanate and iodide, have often been independently tested on Candida suspensions. ${ }^{8,20}$
Hypoiodite decreases the survival rate at a lower concentration than hypothiocyanite. ${ }^{20}$ However, the hypohalous ion present in the oral cavity is hypothiocyanite rather than hypoiodite because the substrate $\mathrm{SCN}^{-}$at the origin of the former is 1,000 times more abundant than iodide $\left(\mathrm{I}^{-}\right)$at the origin of the latter: in fact, the salivary thiocyanate results in salivary concentrations in the $\mathrm{mM}$ while iodide scarcely reaches $\mu \mathrm{M}$ concentrations. Mixtures of the two substrates, thiocyanate and iodide, have been less often investigated but seem to result in the formation of a more stable oxidative complex over time. ${ }^{8,14,21}$ Few of the papers claiming that lactoperoxidase activity produces a $\mathrm{OSCN}^{-} / \mathrm{OI}^{-}$mixture as products in the presence of an $\mathrm{SCN}^{-} / \mathrm{I}^{-}$substrate mixture actually analyzed the products generated under their experimental conditions. ${ }^{5,8,13}$ The relative concentration of the substrates, and the $\mathrm{pH}$ and ionic strength of the media modified the nature of active ions produced by lactoperoxidase and the biological activity of the enzyme. ${ }^{14}$ Under well-defined experimental conditions - ie, 1) high ionic strength, 2) a ratio of KI:KSCN around 4.5, and 3) in vitro enzymatic reaction in a neutral or acid buffer - an iodine-thiocyanate complex is produced. ${ }^{14}$ Indeed, using ${ }^{13} \mathrm{C}$ nuclear magnetic resonance spectroscopy, OSCN ${ }^{-}$is observed only when using $\mathrm{H}_{2} \mathrm{O}_{2}$ and $\mathrm{SCN}^{-}$as substrates, while in the presence of a mixture of $\mathrm{SCN}^{-} / \mathrm{I}^{-}$, the peak of $\mathrm{OSCN}^{-}$is replaced by another corresponding to $\mathrm{I}_{2} \mathrm{SCN}^{-} .{ }^{14}$ In particular, the use of a highly concentrated neutral buffer such as $0.1 \mathrm{M}$ phosphate buffer $\mathrm{pH} 7.4$ optimized the oxidoreduction of iodide and thiocyanate by lactoperoxidase and yielded more concentrated ion solutions in the form of $\mathrm{I}_{2} \mathrm{SCN}^{-}$, which showed in vitro inhibition of $P$. expansum, a fungus responsible for various fruit pathologies. ${ }^{14}$ The formation of $\mathrm{I}_{2}(\mathrm{SCN})^{-}$iodine-thiocyanate complex has already been incriminated in older studies when mixing $\mathrm{I}_{2}$ or $\mathrm{I}^{-}$with SCN. ${ }^{22-25}$

The oxidant concentrations produced were less toxic to the epithelial cells of the mouth than chlorhexidine. Indeed, $1,000 \mu \mathrm{M}$ oxidant solution increased the percentage of epithelial cell mortality in saliva by $20.0 \%$ on average (from $34.8 \%$ to $54.8 \%$ ) while chlorhexidine increased it by $65.2 \%$ (from $34.8 \%$ to $100.0 \%$ ). When diluted 3 times, the oxidant solution retained its anti-Candida activity while reducing the mortality of the oral cells by $\sim 13.0 \%$. The resistance of epithelial cells can be explained on the one hand by the presence of bacteria covering their surface and forming a protective biofilm, and on the other hand by detoxifying enzymatic activities in some commensal species present in the oral environment. ${ }^{26}$ Rinsing dentures after ex vivo incubation in the oxidant solution will further limit eventual effect 
on epithelial cells. A recent paper described on HeLa cells a beneficial balance between the active concentration and the toxic one for iodine-thiocyanate complexes produced in a non-enzymatic manner, by comparison with other biocide molecules such as hydrogen peroxide. ${ }^{27}$ Further studies will have to analyze the interference between this solution of iodine-thiocyanate complexes and the oral microbial ecology. The efficacy of the iodo-thiocyanate complexes prepared without peroxidase activity has already been demonstrated in vitro on Pseudomonas aeruginosa and on Staphylococcus aureus both in suspension and in biofilms. ${ }^{28}$

\section{Ex vivo denture decontamination}

Denture hygiene is difficult for patients to achieve; Candidacontaminated dentures are a gateway for local and systemic infections. Nevertheless, yeast carriage seemed poorly influenced by the hygiene techniques currently available. According to the information gathered by the anamnesis of all the patients and detailed in Table 1, it appears that patients with a contaminated denture show similar levels of hygiene as other patients, and that regular use of toothpaste and mouthwashes (sometimes with antiseptics) does not prevent the colonization of dentures by Candida. A previous paper showed that commercial denture cleaners have effective in vitro candidacidal activity both in terms of biofilm removal and disinfection, but the persistence of a residual biofilm could lead to continuous recolonization of the prostheses. ${ }^{29}$ Alternative cleaning methods are needed to improve biofilm removal but these cannot be based on the use of antifungal therapy agents due to the development of resistance. The results reported in this ex vivo clinical study clearly show that a solution of iodine-thiocyanate complexes affected Candida carriage in $60 \%$ of the tested dentures after only a 5 -minute incubation. Failures can be explained by a Candida carriage at the initial stage above the upper analytical range, thus precluding documentation of a reduction in the number of colonies. They could also be attributed to the presence of resistant strains. The candidacidal effect was active against the two Candida species observed on the denture studied: C. albicans and C. glabrata. A subsequent clinical investigation should follow a cohort of patients who have decontaminated their dentures every day for several days.

\section{Conclusion}

The poor susceptibility to antifungals of microorganisms incorporated in biofilms and the emergence of strains that are resistant to conventional antifungals should encourage their restriction to the treatment of proven infections. This investigation suggests a promising new strategy that could be used to remove Candida from dentures with the aim of preventing mucosal invasion and oropharyngeal candidiasis, especially in medically compromised patients. The new method proposed herein affects the growth of Candida in liquid medium and limits its presence in biofilms. Other preclinical studies should investigate the influence of iodine-thiocyanate complexes on the whole oral microbiome. The preliminary preparation of the solution and the ex vivo treatment of dentures reduced the catalase competition for hydrogen peroxide in the oral environment and allowed sufficient concentrations of oxidants produced by lactoperoxidase to be reached for yeast inhibition. The experimental conditions of the described procedure promote the production of iodine-thiocyanate complexes by lactoperoxidase that is characterized by a stability compatible with hygiene applications achievable in patients' homes.

\section{Acknowledgments}

This study was supported by a grant from the Xenophilia Funds (Université Libre de Bruxelles, Brussels, Belgium). The authors thank Ch. Hauet and L. Malashkina from the Haute Ecole Francisco Ferrer (Medical Biology section) for their enthusiastic participation in the investigations in the context of their final year dissertation.

\section{Disclosure}

Françoise Bafort and Jean-Paul Perraudin are two of the patented owners of the biological applications of iodinethiocyanate complexes (Bafort F, Perraudin J-P, Jijakli H Composition comprenant des ions $\mathrm{I}_{2} \mathrm{SCN}^{-}$et/ou des ions $\mathrm{I}(\mathrm{SCN})^{-2}$. WO2017046407A1, 2015). The authors report no other conflicts of interest in this work.

\section{References}

1. Clem WH, Klebanoff SJ. Inhibitory effect of saliva on glutamic acid accumulation by Lactobacillus acidophilus and the role of the lactoperoxidase-thiocyanate system. J Bacteriol. 1966;91(5):1848-1853.

2. Smith QT, Yang CH. Salivary myeloperoxidase of young adult humans. Proc Soc Exp Biol Med. 1984;175(4):468-475.

3. Thomas EL, Jefferson MM, Joyner RE, Cook GS, King CC. Leukocyte myeloperoxidase and salivary lactoperoxidase: identification and quantification in human mixed saliva. J Dent Res. 1994;73(2):544-555.

4. Ashby MT. Inorganic chemistry of defensive peroxidases in the human oral cavity. J Dent Res. 2008;87(10):900-914.

5. Pruitt KM, Reiter B. Biochemistry of peroxidase system: antimicrobial effects. In: Pruitt KM, Tenovuo JO, editors. The Lactoperoxidase System: Chemistry and Biological Significance. New York: Marcel Dekker; 1985:143-178.

6. Tenovuo JO. Nonimmunoglobulin defense factors in human saliva. In Tenovuo JO, editor. Human Saliva: Clinical Chemistry and Microbiology. Vol. 2. Boca Raton: CRC Press; 1989:55-119. 
7. Bafort F, Parisi O, Perraudin JP, Jijakli MH. Mode of action of lactoperoxidase as related to its antimicrobial activity: a review. Enzyme Res. 2014;2014:517164.

8. Bosch EH, Van Doorne H, De Vries S. The lactoperoxidase system: the influence of iodide and the chemical and antimicrobial stability over the period of about 18 months. J Appl Microbiol. 2000;89(2):215-224.

9. Lenander-Lumikari M, Tenovuo J, Mikola H. Effects of a lactoperoxidase system-containing toothpaste on levels of hypothiocyanite and bacteria in saliva. Caries Res. 1993;27(4):285-291.

10. Kirstilä V, Lenander-Lumikari M, Söderling E, Tenovuo J. Effects of oral hygiene products containing lactoperoxidase, lysozyme, and lactoferrin on the composition of whole saliva and on subjective oral symptoms in patients with xerostomia. Acta Odontol Scand. 1996;54(6):391-397.

11. Gil-Montoya JA, Guardia-López I, González-Moles MA. Evaluation of the clinical efficacy of a mouthwash and oral gel containing the antimicrobial proteins lactoperoxidase, lysozyme and lactoferrin in elderly patients with dry mouth: a pilot study. Gerodontology. 2008;25(1):3-9.

12. Sebaa S, Lybaert P, Boucherit-Otmani Z, Courtois P, Ahariz M. Ex vivo yeast-decontamination of denture by $\mathrm{H}_{2} \mathrm{O}_{2}$ /iodide/lactoperoxidase system: need to overpass the microbial $\mathrm{H}_{2} \mathrm{O}_{2}$ catabolism. Oral Health Dent Manag. 2015;14(1):62-69.

13. Bafort F, Parisi O, Perraudin J-P, Jijakli H. The lactoperoxidase system, a natural biochemical biocontrol agent for pre- and post-harvest applications. J Phytopathol. 2017;165(1):22-34.

14. Bafort F, Damblon C, Smargiasso N, De Pauw E, Perraudin JP, Jijakli $\mathrm{MH}$. Reaction product variability and biological activity of the lactoperoxidase system depending on medium ionic strength and $\mathrm{pH}$, and on substrate relative concentration. Chem Biodivers. 2018;15:e1700497.

15. Blanco MT, Pérez-Giraldo C, Blanco J, Morán FJ, Hurtado C, GómezGarcía AC. In vitro studies of activities of some antifungal agents against Candida albicans ATCC 10231 by the turbidimetric method. Antimicrob Agents Chemother. 1992;36(4):898-901.

16. Aune TM, Thomas EL. Accumulation of hypothiocyanite ion during peroxidase-catalysed oxidation of thiocyanate ion. Eur J Biochem. 1977;80(1):209-214.
17. Stepanović S, Vuković D, Dakić I, Savić B, Švabić-Vlahović M. A modified microtiter-plate test for quantification of staphylococcal biofilm formation. J Microbiol Methods. 2000;40(2):175-179.

18. Sebaa S, Hizette N, Boucherit-OtmaniZ, Courtois P. Dose-dependent effect of lysozyme upon Candida albicans biofilm. Mol Med Rep. 2017;15(3): 1135-1142.

19. Fadel M, Courtois P. Inhibitory effect of lactoperoxidase-generated hypothiocyanite upon black pigmented anaerobe growth. Int $\mathrm{J} \mathrm{Mol}$ Med. 2001;8(1):59-62.

20. Ahariz M, Courtois P. Candida albicans susceptibility to lactoperoxidase-generated hypoiodite. Clin Cosmet Investig Dent. 2010;2:69-78.

21. Schlorke D, Flemmig J, Birkemeyer C, Arnhold J. Formation of cyanogen iodide by lactoperoxidase. J Inorg Biochem. 2016;154:35-41.

22. Lewis C, Skoog DA. Spectrophotometric study of a thiocyanate complex of iodine. J Am Chem Soc. 1962;84(7):1101-1106.

23. Long C, Skoog DA. A thiocyanate complex of iodine(I). Inorg Chem. 1966;5(2):206-210.

24. Országh I, Bazsa G, Beck MT. Spectrophotometric study of the reversible iodine-thiocyanate interaction. Inorganica Chim Acta. 1972;6:271-274.

25. Scott McIndoe J, Tuck DG. Studies of polyhalide ions in aqueous and non-aqueous solution by electrospray mass spectrometry. Dalt Trans. 2003;158:244-248.

26. Courtois P, Pourtois M. Purification of NADH-hypothiocyanite-oxidoreductase in Streptococcus sanguis. Biochem Mol Med. 1996;57(2): 134-138.

27. Tonoyan L, Boyd A, Fleming GTA, Friel R, Gately CM. In vitro comparative cytotoxicity study of a novel biocidal iodo-thiocyanate complex. Toxicol In Vitro. 2018;50:264-273.

28. Tonoyan L, Fleming GTA, Mc Cay PH, Friel R, O'Flaherty V. Antibacterial potential of an antimicrobial agent inspired by peroxidase-catalyzed systems. Front Microbiol. 2017;8:680.

29. Jose A, Coco BJ, Milligan S, et al. Reducing the incidence of denture stomatitis: are denture cleansers sufficient? J Prosthodont. 2010;19(4): $252-257$.
Clinical, Cosmetic and Investigational Dentistry

\section{Publish your work in this journal}

Clinical, Cosmetic and Investigational Dentistry is an international, peer-reviewed, open access, online journal focusing on the latest clinical and experimental research in dentistry with specific emphasis on cosmetic interventions. Innovative developments in dental materials, techniques and devices that improve outcomes and patient satisfac-

\section{Dovepress}

tion and preference will be highlighted. The manuscript management system is completely online and includes a very quick and fair peerreview system, which is all easy to use. Visit http://www.dovepress. com/testimonials.php to read real quotes from published authors. 